\title{
Autonomy and Severe Mental Illness: The Relationship between Social Network and Functioning
}

\author{
Henrik Levinsson ${ }^{1 *}$, Lars Kjellin² and Margareta Östman ${ }^{3}$ \\ ${ }^{1}$ Department of Psychology, Lund University, Sweden \\ ${ }^{2}$ University Health Care Research Center, Faculty of Medicine and Health, Orebro University, Sweden \\ ${ }^{3}$ Faculties of Health and Society, Malmo University, Sweden
}

Received: October 14, 2016; Accepted: November 15, 2016; Published: November 17, 2016

*Corresponding author: Henrik Levinsson, Department of psychology, Box 213, SE-221 00 Lund, Tel: $00462229714 ;$ Fax: 004622242 09; E-mail: henrik. levinsson@psy.lu.se

\begin{abstract}
Purpose: The aim of the present study was to illuminate a theoretical framework about autonomy as a capacity, which takes into consideration both the cognitive functioning of the individual as well as access to social networks.

Design and Method: In order to illuminate a theoretical framework about autonomy as a capacity, a quantitative study comprising a dataset of involuntarily and voluntarily admitted patients at acute general psychiatric wards was used.

Findings: Respondents who reported meeting friends, family, relatives or acquaintances often had a significant higher functioning in terms of total GAF score compared to respondents who reported meeting friends, family, relatives or acquaintances as seldom or never.

Practice Implications: The efforts of nursing care practices to care for and treat severe mental illness may benefit from greater awareness about research on functioning and access to social networks. Such research is a valuable contribution to further develop a theoretical framework about autonomy within the nursing profession.
\end{abstract}

Keywords: Autonomy; Global Assessment of Functioning; Nursing Practice; Severe Mental Illness; Social Network

\section{Introduction}

In a qualitative study [1] it is indicated that mental health nurses working in acute inpatient settings experience a need for adjustment regarding their role in the assessment process of treatment. A cornerstone in nursing practice is to adopt a holistic patient perspective, taking into account not only the patient's pathology but also psychological and social factors. While a holistic perspective is important in the care of patients, there is also the need for conceptual development of patient autonomy within nursing practice since the meaning of autonomy is shown to vary [2]. A wider knowledge and discussion among nurses about the meaning of autonomy might result in an enhanced nursing profession which also might lead to that a more solid theoretical framework can be developed.
Autonomy is one of the central concepts in the provision of nursing and health care practices [3]. The most common understanding of autonomy is self-rule, or self-determination [4]. However, no consensus among nurses exists about how autonomy should be interpreted [2] and it is not always clear whether autonomy is referred to as a right to be respected, or a capacity of the individual [4].

As a right, autonomy refers to a fundamental ethical principle commonly applied in the health care system, namely, the principle of autonomy. This principle aims to protect the patient's right to make decisions affecting her or his health and care [3]. Respecting the patient's right to make own decisions with reference to the principle of autonomy is a cornerstone in everyday nursing practice.

Autonomy as a capacity refers to the ability to rule over oneself. For instance, in a health care context this could mean that patients are treated as autonomous if they are assessed to have enough insight into their medical condition. As autonomous patients they are also expected to be able to manage relevant information about various treatment alternatives provided by clinicians, as well as being able to evaluate the consequences if acting upon them [4].

Further on, this paper will illuminate autonomy as a capacity of the individual with a focus on severe mental illness.

\section{Autonomy and Severe Mental Illness}

The capacity to be autonomous may be understood as a set of psychological functions of the individual. These can vary in degree and over time depending on contextual factors, and so a person can be more or less autonomous [3,4]. Different forms of severe mental illness can result in decreased functioning and hence reduced autonomy. For instance, there might be reduced autonomy with thought insertion, a psychotic symptom in which a person perceives his or her thoughts and actions as stemming from the outside and being controlled by somebody else. In this delusional state the person does not experience those thoughts as her own. Since patients suffering from thought 
insertion commonly feel alienated from their own thoughts and preferences, it does not seem appropriate to ascribe a person in this state any great degree of autonomy, and certainly not with regard to the content of these thought intrusions. More examples of reduced autonomy may be displayed in severe depressive episodes and manic phases of bipolar disorder.

As part of the mental health care deinstitutionalization processes which highlight the right to participate in society just as everyone else, and to make decisions for oneself in society, it is also reasonable to claim that there is an increased demand (or even an expectation) to be one's own person, that one is autonomous [4]. However, severe mental illness could result in considerable impairments in everyday functioning [5-7], and from a cognitive perspective, research has found that patients with schizophrenia have a reduced metacognitive capacity [8] as well as difficulties with metacognitive agency [9].

Importantly, the understanding of autonomy as a capacity also comprises the surrounding context of the individual. From this perspective, assessment tools measuring the level of functioning and symptoms, like Global Assessment of Functioning (GAF) [10], may capture central aspects of what it means to be autonomous. However, social functioning can be difficult to measure [11] and to our knowledge no golden standard of how to evaluate it seems to exist. Compared to social and occupational functioning, clinical factors have been shown to be the primary determinants in order to predict a patient's total GAF score [12].

Many persons with severe mental illness are shown to have a reduced social functioning, have poor social networks and live in isolation $[7,13]$. However, supported socialization has been found to increase functioning, self-esteem and social networks among persons with mental illness $[7,14]$. In light of these findings, autonomy as a capacity should not solely be understood in terms of internal cognitive factors, but also take into consideration the social surrounding of the individual. From this perspective, autonomy might be seen as relational $[15,16]$.

The aim of the present study is to illuminate a theoretical framework about autonomy as a capacity, which takes into consideration both the cognitive functioning of the individual as well as access to social networks. In order to test the theoretical framework, we decided to use an earlier collected data set, comprising information about total GAF score as well as access to social networks among persons with severe mental illness.

To illustrate the theoretical framework about autonomy as a capacity we hypothesized that patients who meet friends more often also have a higher functioning.

\section{Methods}

\section{Design}

In our empirical analysis we used a data set gathered in the late 1990s. The data set is the Swedish part of a Nordic study of voluntary and in voluntary psychiatric inpatient care [17].

The data set included a consecutive sample of involuntarily committed patients and a randomly selected sample of voluntarily admitted patients at acute general psychiatric wards. Excluded in the Nordic study were people aged under 18 and over 70, and people with a primary diagnosis of alcohol or substance abuse, with severe learning disabilities or severe dementia, or not speaking Swedish.

In the original study, informants were contacted and asked if they wanted to participate in the study within the first week of admission. All interviews were conducted by a psychiatrist, psychologist, social worker, or nurse trained in conducting interviews for the study. Follow-up interviews were performed, but the present article is based on data from the first interview only. None of the interviewers were involved in providing treatment to the patients they interviewed. Throughout the study concordance in ratings between interviewers were checked using videotaped training interviews.

\section{Settings}

The study was conducted at inpatient psychiatric services of four different Swedish centers, each of which had comprehensive responsibility for a geographically defined catchment area of both urban and rural composition with a total population from 90,000 to 260,000 inhabitants. The psychiatric departments in the four counties during the period of investigation had 3.13-5.13 beds/ 10,000 inhabitants allocated for short-term psychiatric care.

\section{Participants}

Of 375 patients asked to participate in the Nordic study, 138 involuntary and 144 voluntary patients (in all 282) accepted. Involuntarily admitted patients declined more often than voluntarily admitted ( $30 \%$ vs. $\left.20 \%, \chi^{2}=5.056, d f=1, p=.025\right)$. Of included patients, $63.5 \%$ were women and the mean (sd) age was 42.2(12.7), which did not differ from the distribution on gender and age among all eligible patients. The mean (sd) total GAF score was 37.0(13.1). The distribution on diagnostic groups according to the Diagnostic and Statistical Manual of Mental Disorders, Fourth Edition [10] were $36.7 \%$ psychoses (schizophrenia, delusional disorders, schizoaffective and schizophreniform disorders, and atypical psychoses), 37.0 \% affective mood disorders and 26.3\% other diagnoses (e.g. personality and anxiety disorders).

Due to missing values, the analyses in the present study are based on 249-268 cases.

\section{Instruments}

To measure level of functioning, the Global Assessment of Functioning [10] was used. The current level of functioning at the time of assessment was rated and recorded as a single total GAF score. GAF has proven validity and shows a relatively high interrater reliability $[18,19]$. With GAF a general external assessment of functioning together with assessment of symptoms was made, resulting in a total GAF value between 1 and 100 in which lower values indicate a lesser degree of functioning and vice-versa.

Patient interviews were structured and the interviewer classified the answers into fixed categories. The interview protocol has been used in earlier studies where the inter rater 
reliability was found to be good with a mean Cohen's kappa value for all interview questions of .96 [20]. Access to social network was captured by one question in the interview; how often do you meet friends, family, relatives or acquaintances? Answers were classified into often (twice a month or more) or seldom (once a month, less than once a month, or never).

\section{Ethical considerations}

All persons included in the original study were provided with oral and written information about the study through a contact person known to them and through the interviewer. Those who agreed to take part in the investigation gave their informed consent prior to the interview, after the study had been fully explained to them. The study was approved by the Research Ethics Committee of the Medical Faculty, University of Uppsala, Sweden (registration number 96408).

\section{Statistical Analysis}

Data were analyzed using IBM SPSS Version 22. In order to compare total GAF scores between groups, independent sample t-tests were used and $p$-values $<.05$. were considered significant.

\section{Results}

In the original study, 227 (85\%) of the patients reported meeting friends, family, relatives or acquaintances often.

There was a significant difference in total GAF scores between respondents who reported meeting friends, family, relatives or acquaintances often and those whose answers were classified as seldom or never: mean (sd) GAF 38.7 (12.3) vs. 31.1 (12.1); t $(266)=3.69, p=.001$.

\section{Discussion}

Reduced social functioning, poor social networks and isolation are common among persons living with a severe mental illness [7,13], and aspects concerning functioning, access to social network and autonomy are issues for research.

The present study sought to illuminate a theoretical framework of autonomy as a capacity, measured by total GAF score, and understood as relational, i.e. in relation to access to social network. The difference found in total GAF scores according to different access to social network might indicate some support for our hypothesis, although no strong conclusions about functioning and access to social network can be drawn based on our data.

The aim of our study was not to not reveal any strict causal relationship between total GAF score and access to social network, or any direction of that relationship. Thus, the findings do not make known if higher functioning according to GAF implies that one more often meets with the social network, or whether meeting the social network more often signifies higher functioning. Individuals who already have relatively high functioning may possess considerably more friends than those assessed to have a low degree of functioning.

Phenomenon like social network can be difficult to evaluate and measure within research. An important distinction to be made concerning the operationalization of social network is between quality and quantity. For example, having a large network does not necessarily mean that one experiences a great deal of social support. One can still have feelings of loneliness. Objectively, a person can have numerous friends but not perceive this to be so. On the other hand, a person might have a very limited access to social network and at the same time regard it as qualitatively rich.

\section{A Theoretical Framework About Autonomy as a Capacity}

Being autonomous may evoke images about relying on the person's own capacity in order to manage her life, and that an autonomous person is to some extent independent. Empirical research, on the other hand, indicates that the access to social network might enable functioning $[7,14]$.

Autonomy should not solely be understood as a right. Autonomy also refers to a capacity of the individual. This distinction is valuable and important to communicate in both educational supports for professionals and health care services. As shown in the present study, a patient's autonomy might be associated with her access to social network and in this way, to some extent regarded as relational.

Thus, the principle of autonomy does not exclusively pertain to blindly defending the patient's right to have her autonomy respected. An ethical problem arises in cases where autonomy is expected, but when in fact the patient's capacity in this regard is limited. Such a person might be put at risk if left to take care of her in the absence of access to a social network.

One of the presuppositions could be that establishing access to a social network enables the possibility of enhancing autonomy. However, before starting to implement methods of improving social network and social support, the efficacy of such interventions requires further investigation [21,22].

\section{Methodological Considerations}

In the present paper we have tried to illuminate and develop the concept of autonomy as a capacity. Theoretical development of central but complex concepts, such as autonomy, is in line with the nursing profession and might be important in both research and education.

There are shortcomings in terms of ecological validity in using data sets of old age for analyses as is done in this study. However, it is also valuable to use already gathered information from, in this case, persons with a severe mental illness in order to avoid taking more of their valuable time than needed.

GAF is one tool used in research in order to try to assess functioning. GAF can be claimed to be a reliable instrument, but is not regarded as a golden standard by which to assess functioning. This is a possible weakness of the present study but to our knowledge, accessible tools for measuring aspects of autonomy in terms of functioning are rather limited. In DSM-5 [23] GAF is deleted and there is a need for other measurement 
tools that capture central aspects of functioning. However, for present purposes we judged GAF to be appropriate to use in order to underline our reasoning about autonomy as a capacity.

Our choice of comparing total GAF score as a measure for functioning with a question measuring access to social network might be questioned since the latter may have been taken into account in the GAF assessments. However, in our opinion qualitative aspects of social functioning are assessed with GAF while the question about access to social network measures quantitative aspects.

\section{Future Research and Implications for Nursing Practice}

To further examine the association between autonomy as a capacity and access to social network, we welcome more updated studies. It would also be of great importance to conduct longitudinal studies in order to obtain greater knowledge about functioning and access to social network over time. Such studies would hopefully also contribute to a better understanding within the whole health care system of what it means for an individual with a severe mental illness to live an autonomous life "in society" and the challenges associated with it.

Research on functioning and access to social network indicates the need for mental health care to develop strategies to strengthen patients' social network and autonomy, as well as to refine tools to measure these phenomena. Future interventions during subsequent care of both inpatients and outpatients might focus on the effect of having limited access to social networks.

Our aim with developing a theoretical framework about autonomy as a capacity is in line with the idea that nursing practice should be seen as a relational and intersubjective process, focusing on the relation between nurse and patient $[24,25]$. From this perspective, the present study can be seen as a contribution to the development of everyday nursing practice concerning the approach to patient autonomy.

\section{Acknowledgement}

No financial grants and other funding are related to the project.

\section{Conflict of Interest}

No conflict of interest and no author report any financial or other conflict of interest regarding this work.

\section{Ethical Approval}

The study was approved by the Research Ethics Committee of the Medical Faculty, University of Uppsala, Sweden (registration number 96408).

All persons included in the study were provided with oral and written information about the study through a contact person known to them and through the interviewer. Those who agreed to take part in the investigation gave their informed consent prior to the interview, after the study had been fully explained to them.

\section{References}

1. Bishop D, Ford-Bruins I. Nurses' perceptions of mental health assessment in an acute inpatient setting in New Zealand: A qualitative study. Int J Ment Health Nurs. 2003;12(3):203-212.
2. Lindberg C, Fagerström C, Sivberg B, Willman A. Concept analysis: patient autonomy in a caring context. J Adv Nurs. 2014;70(10):22082221. doi: 10.1111/jan.12412.

3. Beauchamp TL, Childress JF. Principles of biomedical ethics. 5th ed New York: Oxford University Press. 2001.

4. Levinsson H. Autonomy and metacognition: A health-care perspective. Lund University. 2008.

5. Harvey PD. Mood symptoms, cognition, and everyday functioning: in major depression, bipolar disorder, and schizophrenia. Innov Clin Neurosci. 2011;8(10):14-18.

6. Iosifescu DV. The relation between mood, cognition and psychosocial functioning in psychiatric disorders. Eur Neuropsychopharmacol. 2012;22:499-504. doi: 10.1016/j.euroneuro.2012.08.002.

7. Sheridan AJ, Drennan J, Coughlan B, O’Keeffe D, Frazer K, Kemple $\mathrm{M}$, et al. Improving social functioning and reducing social isolation and loneliness among people with enduring mentalillness: Report of a randomised controlled trial of supported socialisation. Int J Soc Psychiatry. 2015;61(3):241-250. doi: 10.1177/0020764014540150.

8. Kircher TT, Koch K, Stottmeister F, Durst V. Metacogniton and reflexivity in patients with schizophrenia. Psychopathology. 2007;40(4):254-260.

9. Metcalfe J, Van Snellenberg JX, DeRosse P, Balsam P, Malhotra AK. Judgements of agency in schizophrenia: an impairment in autonoetic metacognition. Philos Trans R Soc Lond B Biol Sci. 2012;367(1594):1391-400. doi: 10.1098/rstb.2012.0006.

10. American Psychiatric Association. Diagnostic and statistical manual of mental disorders. 4th ed Washington DC: APA. 1994.

11. Suzuki T, Uchida H, Sakurai H, Ishizuki T, Tsunoda K, Takeuchi H, et al. Relationships between global assessment of functioning and other rating scales in clinical trials for schizophrenia. Psychiatry Research. 2015;227(2-3):265-269. doi: 10.1016/j.psychres.2015.02.024.

12. Urbanoski KA, Henderson C, Castel S. Multilevel analysis of the determinants of the globalassessmentof functioning in an inpatient population. BMC Psychiatry. 2014;14(63). doi:10.1186/1471244X-14-63.

13. Nilsson LL, Logdberg B. Dead and forgotten-postmortem time before discovery as indicator of social isolation and inadequate mental healthcare in schizophrenia. Schizophr Res. 2008;102(1-3):337-339. doi: 10.1016/j.schres.2008.03.011.

14. Perry BL, Pescosolido BA. Social network activation: The role of health discussion partners in recovery from mental illness. SocialScience \& Medicine. Soc Sci Med. 2015;125:116-128. doi: 10.1016/j. socscimed.2013.12.033.

15. Levinsson H, Östman M, Kjellin L. Relational Autonomy and Psychiatry. In: The $50^{\text {th }}$ annual convention of the AISB; 2014 Apr 1-4; Goldsmiths University of London. 2014:67.

16. Walter JK, Ross LF. Relational Autonomy: Moving Beyond the Limits of Isolated Individualism. Pediatrics. 2014; 133:16-23. doi: 10.1542/ peds.2013-3608D.

17. Kjellin L, Andersson K, Bartholdson E, Candefjord IL, Holmstrøm H, Jacobsson L, et al. Coercion in psychiatric care - patients' and relatives' experiences from four Swedish psychiatric services. Nord J Psychiatry. 2004;58(2):153-159.

18. Söderberg P, Tungström S, Armelius BA. Reliability of global assessment of functioning ratings made by clinical psychiatric staff. Psychiatric Services. 2005;56(4):434-438. doi: 10.1176/appi. ps.56.4.434. 
19. Tungström S, Söderberg P, Armelius BA. Relationship between the Global Assessment of Functioning and other DSM axes in routine clinical work. Psychiatric Services. 2005;56(4):439-443. doi: 10.1176/ appi.ps.56.4.439.

20.Candefjord IL, Lampinen K, Ängfors G. Patienters upplevelse av akut psykiatrisk vård. (Patients' experiences of short-term inpatient psychiatric care. In Swedish). Socialmedicinsk Tidsskrift. 1994;71:447-453.

21. Hogan BE, Linden W, Najarian B. Social support interventions - Do they work? Clinical Psychology Review. Clin Psychol Rev. 2002;22(3):383442.
22. Sibitz I, Amering M, Unger A, Seyringer ME, Bachmann A, Schrank B, et al. The impact of the social network, stigma and empowerment on the quality of life in patients with schizophrenia. European Psychiatry. 2011;26(1):28-33. doi: 10.1016/j.eurpsy.2010.08.010.

23. American Psychiatric Association. Diagnostic and statistical manual of mental disorders. 5th ed Washington DC: APA. 2013.

24.D'Antonio P, Beeber L, Sills G, Naegle M. The future in the past: Hildegard Peplau and interpersonal relations in nursing. Nursing Inquiry. Nurs Inq. 2014;21(4):311-7. doi: 10.1111/nin.12056.

25. Peplau H. Interpersonal relations in nursing, a conceptual frame of reference for psychodynamic nursing. New York: Putnam. 1952. 